\title{
Examine a dynamic of mother-to-child transmission of HIV in the population-based surveys
}

\author{
Rathavuth Hong $^{1^{*}}$, Rathnita Them ${ }^{2}$ \\ ${ }^{1}$ Measure Demographic and Health Survey, ICF Macro, Calverton, USA; \\ *Corresponding Author: rhong@icfi.com \\ ${ }^{2}$ College of Health and Human Services, George Mason University, Fairfax, USA.
}

Received 29 June 2011; revised 23 September 2011; accepted 14 October 2011.

\begin{abstract}
The main source of HIV infection in young children is of their mothers, during pregnancy, labor and delivery, or by breastfeeding. The rate of HIV transmission from infected mothers to their newborn children varies from $15 \%$ to $40 \%$ with one-thirds of these infections was through breastfeeding. This paper examines cross-sectional population-based survey data of HIV test results among mothers and their children in Uganda, Swaziland to estimates of mother-tochild transmission (MTCT) rate of HIV infection. The prevalence of HIV among women aged 15 49 who gave birth in the past 5 years in Uganda is $7.3 \%$, and in Swaziland is $37.9 \%$. The HIV prevalence of children who mothers were HIV positive were very similar: $\mathbf{1 0 . 3 \%}$ in Uganda and $11.5 \%$ in Swaziland. This association represents the crude rate of MTCT in these two countries at the time of the survey. Presence of HIV antibody in early age ( 0 - 11 months) is due to both true infection and passive antibody from mothers. The seroconversion dropped nearly half in the second year of age which was likely that many of these children died before reaching the age of 12 - 23 months and passive antibody were gradually cleared at this point. This analysis demonstrated that cross-sectional data can be used to estimate indirectly the magnitude and dynamic of MTCT.
\end{abstract}

Keywords: HIVIAIDS; Mother-to-Child;

Transmission; Demographic and Health Survey; Uganda; Swaziland

\section{INTRODUCTION}

The source of HIV infection in young children is overwhelming of their mothers, during pregnancy, labor and delivery, or by breastfeeding. In the region where the rate of HIV infection among pregnant women is very high, for example exceeds 35\%, HIV/AIDS infection contributes to as high as $42 \%$ of child mortality [1]. In developing countries, the rate of HIV transmission from infected mothers to their newborn children varies from study to study, and is estimated from about $15 \%$ to $40 \%$. Among those children who are infected HIV through the transmission from their mothers, one-third received the virus through breastfeeding [2].

We examine whether data on HIV testing in the cross-sectional data such as population-based survey provide good estimates of mother-to-child transmission (MTCT) rate of HIV infection. The testing procedure used two ELISAs parallel testing algorithm according to WHO testing algorithm. All discordant samples were subjected to a second round of testing using both tests. The discordances from the second round are "indeterminate" and were then subjected to a third confirmatory Western-Blot test. The Western-Blot result was considered final for the indeterminate samples.

\section{METHODS}

\subsection{Data}

We used data from the 2004-2005 Uganda HIV/AIDS Sero Behavioural Survey (UHSBS) and from the 2006 Swaziland Demographic and Health Survey (SDHS). These are national representative household surveys that include HIV testing among children and adults. UHSBS tested HIV among 8,374 children aged 0 - 59 months, 10,227 women age 15 - 59 years, and 8,298 men age 15 59 years in a random sample of 9,529 households across the country. Information on birth history from the women questionnaire was used to establish the link between mothers and her biological children for the analysis of this study.

\subsection{Statistical Methods}

Statistical analysis was performed using Stata 10.0 
Table 1. Association of children's HIV status with mother's HIV status, Uganda AIS 2004-2005 and Swaziland DHS 2007.

\begin{tabular}{|c|c|c|c|c|}
\hline \multirow[b]{2}{*}{ Variable } & \multicolumn{2}{|c|}{ Uganda (0 - 59 months) } & \multicolumn{2}{|c|}{ Swaziland (24 - 59 months) } \\
\hline & Percentage HIV positive & Number of children & Percentage HIV positive & Number of children \\
\hline \multicolumn{5}{|l|}{ Mother’s HIV status } \\
\hline Negative & 0.14 & 6607 & 0.79 & 345 \\
\hline Positive & 10.25 & 364 & 11.52 & 176 \\
\hline Not tested/missing & 0.76 & 1403 & 6.57 & 306 \\
\hline Total & 0.68 & 8374 & 5.20 & 827 \\
\hline
\end{tabular}

Table 2. Association of children's HIV status with mother's HIV status, by age of the child, Uganda AIS 2004-2005.

\begin{tabular}{|c|c|c|c|c|c|}
\hline \multirow[b]{2}{*}{ Child's age (month) } & \multicolumn{4}{|c|}{ Mother HIV's status } & \multirow{2}{*}{ Number } \\
\hline & HIV negative & HIV positive & Unknown, died & Unknown, survived & \\
\hline $0-11$ & 0.00 & 19.44 & 0.00 & 3.02 & 1631 \\
\hline $12-23$ & 0.47 & 10.24 & 0.00 & 0.00 & 1484 \\
\hline $24-35$ & 0.26 & 14.52 & 6.33 & 0.46 & 1667 \\
\hline $36-47$ & 0.00 & 6.37 & 6.35 & 0.43 & 1742 \\
\hline $48-59$ & 0.00 & 2.51 & 3.35 & 0.37 & 1849 \\
\hline Total & 0.14 & 10.25 & 4.40 & 0.54 & 0.68 \\
\hline Number & 6607 & 364 & 80 & 1323 & 8374 \\
\hline
\end{tabular}

(Stata Corp., College Station, TX). Bivariate analyses were conducted to identify the relationship of the HIV-positive status between the children and the mothers at the time of the survey, and the HIV prevalence among the children by age group.

\section{RESULTS}

In the 2004-2005 Uganda HIV/AIDS Sero-Behavioural Survey (UHSBS), 8,374 children age 0 - 59 months from a random sample of 9,529 households were tested for HIV virus with valid test results [3]. The prevalence of HIV infection in women aged 15 - 49 is $7.5 \%$, in women aged 15 - 49 who gave birth in the 5 years before the survey is $7.3 \%$, and in children age 0 59 months was $0.7 \%$. In the 2006 Swaziland Demographic and Health Survey (SDHS), only 827 children age 24 - 59 months were tested for HIV [4], of whom $5.2 \%$ were positive. In Swaziland the HIV prevalence is 31.1 percent among all women aged 15 - 49: 37.9\% among women who gave birth in the last 5 years and 26.0\% among those who did not.

In UHSBS, $83 \%$ of HIV tested children, their biological mothers were also tested for HIV with valid test results; $16 \%$ of the children, their mothers were not tested or results were missing, or they were absent at the time of testing; and about 1 percent of the children, their mothers died before the survey. In Swaziland, 63\% of HIV tested children; their biological mothers were also tested for HIV with valid test results available. In spite of differences in prevalence of adult women and children less than 5 founded in the two surveys, the HIV prevalence of children who mothers were HIV positive were very similar: $10.3 \%$ in Uganda and $11.5 \%$ in Swaziland. Results in Table 1 show strong relationship between HIV positive status of the children and HIV positive status of their biological mother. Nonetheless, there were a small number of children $(0.14 \%$ in Uganda and $0.79 \%$ in Swaziland) who were tested positive for HIV, whereas their biological mothers were tested negative for HIV. This observed discordance could caused by false positive (among the children) and false negative (among the mothers); or by HIV infection afterbirth in children, for example by unsterile or used syringes and needles. The association between HIV positive mother and HIV positive children represents the crude rate of MTCT in these two countries at the time of the survey. At the time of study the preventive MTCT (PMTCT) programs were implemented only in some part of these two countries. However, data on breastfeeding and on PMTCT program is not available in these surveys; and attrition of breastfeeding and impact of PMTCT on the transmission rate are unknown. 


\section{DISCUSSION}

In these cross-sectional data, it is usually not possible to establish a longitudinal association of HIV infection in children and their mothers. Nonetheless, in Uganda where the sample is quite large and includes children age from 0 - 4 years old, the HIV test results among these children can be estimated by age group of $0-11,12-23$, 24 - 35, 36 - 47, 48 - 59 months (age in single month is not available) in order to understand some dynamic of the infection.

About $19.4 \%$ of children aged 0 - 11 months of the HIV positive mothers were tested positive. Presence of HIV antibody in early age is due to both true infection and passive antibody from mothers. The seroconversion among children aged 12 - 23 months dropped nearly half to only $10.2 \%$. It was very likely that these children had the same positive rate as those aged 0 - 11 months, when they were at that age group. Therefore this drop in seroconversion could be a result of (1) many infected children were died before reaching the age of $12-23$ months and (2) children were tested positive due to passive antibody from the mothers were gradually cleared of this passive antibody. Study elsewhere also indicates that children who are HIV positive after birth, about $23 \%$ became seronegative when they are nine months old [5]. The proportion of children aged 24 - 35 months tested positive increased to $14.5 \%$, indicating that some children newly infected at this age, most likely through breast feeding. The prevalence decreased to $6.3 \%$ then to 4.4\% among children age 36 - 47 months and 48 -59 months respectively as infected children continued to die and exit the cohort. It is very unlikely that the passive antibody presence after 24 months of age.

The lack of information on breastfeeding and PMTCT program dwindle the ability to analyze the attrition of breastfeeding and the impact of PMTCT on MTCT of HIV virus; and the lack data on age in single month impede the prospect of decomposing positive rate by smaller age group or performing survival analysis of infected children. Nonetheless, this analysis demonstrated that cross-sectional data can be used to estimate indirectly the magnitude and dynamic of MTCT.

\section{REFERENCES}

[1] Walker, N., Schwärtlander, B., Bryce, J. (2002) Meeting international goals in child survival and HIV/AIDS. Lancet, 360, 284-289. doi:10.1016/S0140-6736(02)09550-8

[2] Mclntyre, J.A. (1999) HIV in pregnancy: A review. Occasional Paper No. 2, World Health Organization, Geneva.

http://who.int/reproductive-health/publications/archive/rh r_99_15/index.html

[3] Ministry of Health (MOH) [Uganda] and ORC Macro. (2006) Uganda HIV/AIDS sero-behavioural survey 2004-2005. Ministry of Health and ORC Macro, Calverton, USA.

[4] Central Statistical Office (CSO) and Macro International Incorporated. (2008) Swaziland demographic and health survey 2006-2007. Mbabane, Swaziland.

[5] Italian Multi-Centre Study (1988) Epidemiology, clinical features, and prognostic factors of paediatric HIV infection. Lancet, ii, 1043-1046. 